\title{
IN TRANSFER PROBLEMS
}

\author{
by \\ C. Magnan \\ Institut d'Astrophusique \\ Paris
}

I have not prepared any formal communication. Nevertheless I would like to mention the types of calculation that are now in progress in Paris.

I am handling transfer problems in moving atmospheres by using Monte Carlo techniques. A twolevel atom and an isothermal atmosphere are always assumed. Two types of geometry are considered: a spherical atmosphere with a constant velocity of expansion, or $a$ flat disk with constant velocities of expansion and rotation. Non-coherent scattering (either entirely incoherent or partially coherent) is assumed with respect to the "local frequency" (as seen by the absorbing atoms).

Concerning the "creation" of photons, two problems have been handled: the photons are created via absorption of a continuous spectrum produced by an underlying photosphere (the Schuster problem) or they are created within the envelope via electronic collision or recombination from ionized states.

The most typical results concern the Schuster

problem. P Cygni type profiles are very well reproduced with the adopted models; symmetrical emission features on both sides of a central absorption line are found in the case of a rotating disk.

At the present time we are limited to cases where the mean number of scatterings is of the order of twenty. The order of magnitude of this number is given by $\tau / V$ (where $\tau$ is the optical depth of the envelope and $V$ the macroscopic velocity). 